\title{
Distance-based maximum likelihood estimation method for node localization in wireless sensor networks
}

\author{
Jing $\mathrm{Xu}{ }^{1, \mathrm{a}}$, Jingsha $\mathrm{He}^{2, \mathrm{~b}}$, Yuqiang Zhang ${ }^{1, \mathrm{c}}$, Fangbo Cai ${ }^{2, \mathrm{~d}}$ \\ ${ }^{1}$ College of Computer Science, Beijing University of Technology, Beijing 100124, China \\ ${ }^{2}$ School of Software Engineering, Beijing Engineering Research Center for loT Software and \\ Systems, Beijing University of Technology, Beijing 100124, China \\ ahxj1108@emails.bjut.edu.cn bjhe@bjut.edu.cn, ${ }^{\mathrm{c}}$ \\ Yuqzhang@emails.bjut.edu.cn, Incaifangbo@sina.com
}

\begin{abstract}
Keywords: Wireless sensor networks; Node localization; Distance-based maximum likelihood estimation
\end{abstract}

\begin{abstract}
Node localization is an important supporting technique in wireless sensor networks (WSNs). The traditional maximum likelihood estimation localization method (MLE) assumes that measurement errors are independent of the distance between anchor node and target node. However, the assumption may contradict with the physical characteristic of some existing measurement techniques, such as the widely-used received signal strength indicator. To address this issue, we propose a distance-based MLE considering the dependence of measurement errors on the distance. The proposed distance-based MLE is formulated as a complicated nonlinear optimization problem. An exact solution method is presented based on first order optimal conditional and to improve the search efficiency, a two-dimensional search method is also given. Simulation experiments are performed to demonstrate the effectiveness of this localization. The simulation results show that the distance-based localization method has better localization accuracy compared with other range-based localization methods
\end{abstract}

\section{Introduction}

In WSNs, the anchor node has its own localization function and can obtain its location by itself. Unknown nodes access their location information with the help of the anchor node [1]. Specifically, an unknown node needs to calculate its location information through communication with the anchor nodes based on some localization techniques. Note that there are some applications that the unknown node may not rely on the anchor nodes for coarse localization [2].

Localization methods can be divided into range-based and range-free method [3]. Range-based location method measures the absolute distance or azimuth information between the anchor node and unknown node, and then calculates the unknown nodes location [4]. Range-free localization method does not need to measure the absolute distance or azimuth information. It estimates the distance between nodes based on the inter-node connectivity, the plurality of routing information exchange, and the overlapping areas [5]. Although the range-free location method has lower requirements for hardware and power consumption, its localization accuracy is lower than the range-based method [6]. Thus in this paper, we focus on the range-based method.

Range-based localization method has relatively higher localization accuracy. It uses one or several distance measurement technologies to estimate the localization of the unknown node [7]. The existing techniques of distance measurement includes the received signal strength indicator (RSSI)time of arrival (TOA) time difference of arrival (TDOA) and angle of arrival (AOA).

Based on the RSSI, the traditional maximum likelihood estimation localization method (MLE) assumes that measurement errors are independent of the distance between anchor node and the unknown target node, and calculate the positon of the unknown target node by solving a convex optimization problem. However, the assumption may contradict with the physical characteristic of 
RSSI. To address this issue, we propose a distance-based MLE considering the dependence of measurement errors on the distance. The proposed distance-based MLE is formulated as a complicated nonlinear optimization problem. To solve the resulted optimization problem, two solution methods have been proposed. The first one is based on the first order optimal condition and the second is based on a two-dimensional search. Simulation experiments are performed to demonstrate the effectiveness of this localization. The simulation results show that the distance-based localization method has better localization accuracy compared with other range-based localization methods.

\section{Distance-based maximum likelihood estimation}

MLE method has improved the accuracy of the range-based localization in a certain extent; however, it does not consider the effect of measurement distances on the measurement errors. To address this issue, this paper proposes an improved distance-based maximum likelihood estimation (DB-MLE) localization method which considers the dependence of measurement errors on the distance.

Specifically, DB-MLE assume that the measurement error will increase as the distance between the anchor node and the unknown node increases. According to this assumption, this method introduces the concept of the measurement error factor which weighs the importance of different measurement values. By introducing this concept, DB-MLE can reduce the effect of the measurement error on localization results, and further improve the localization accuracy.

\subsection{Assumptions and model}

For a given anchor node $P_{M}\left(x_{M}, y_{M}\right)$ and an unknown node $P(x, y)$, let us define the true distance between them as $d_{M}=\sqrt{\left(x-x_{M}\right)^{2}+\left(y-y_{M}\right)^{2}}$. Due to measurement noises, the actual measured distance $r_{M}$ is a random variable which depends on the real value $d_{M}$. Based on the ranging principle, we make the following assumptions:

The measurement distance is proportional to the true distance, satisfying the following equation:

$r_{M}=d_{M}(1+\varepsilon)$. Where the random variables $\varepsilon$ is the Gauss white noise, subject to the normal distribution $N\left(0, \sigma^{2}\right)$.Different measurement distances among different nodes are independent with each other.Under above assumptions, the joint probability density function is given as $f\left(r_{1}, \cdots, r_{N}\right)=\prod_{i=1}^{N} \frac{1}{\sqrt{2 \pi} \sigma d_{i}} \exp \left\{-\frac{\left(r_{i}-d_{i}\right)^{2}}{2 \sigma^{2} d_{i}^{2}}\right\}$, Where the measurement distance between the anchor nodes $P_{i}\left(x_{i}, y_{i}\right)$ and the unknown node $P(x, y)$ is given as $d_{i}=\sqrt{\left(x_{i}-x\right)^{2}+\left(y_{i}-y\right)^{2}}$, and the marginal probability density function is given as $f_{i}\left(r_{i}\right)=\frac{1}{\sqrt{2 \pi} \sigma d_{i}} \exp \left\{-\frac{\left(r_{i}-d_{i}\right)^{2}}{2 \sigma^{2} d_{i}{ }^{2}}\right\}$. The corresponding likelihood function is $\ln f\left(r_{1}, r_{2}, \cdots, r_{N}\right)=-\sum_{i=1}^{N} \ln d_{i}-\sum_{i=1}^{N} \frac{\left(r_{i}-d_{i}\right)^{2}}{2 \sigma^{2} d_{i}^{2}}-N \ln \sqrt{2 \pi} \sigma$.

DR-MLE calculates the position of the unknown node by maximizing the above objective function. Due to its non-convexity, we propose two different methods to solve this problem.

\subsection{First order optimality method}

Note that solve DR-MLE is equivalent to minimize the following objective function: $f(x, y)=\ln \sqrt{(x-a)^{2}+(y-b)^{2}}+\frac{\left(r-\sqrt{(x-a)^{2}+(y-b)^{2}}\right)^{2}}{2 \sigma^{2}\left[(x-a)^{2}+(y-b)^{2}\right]}$. And its derivative is given as: 


$$
\begin{aligned}
& f_{x}=\frac{x-a}{(x-a)^{2}+(y-b)^{2}}+\frac{r(x-a)\left(\sqrt{(x-a)^{2}+(y-b)^{2}}-r\right)}{\sigma^{2}\left[(x-a)^{2}+(y-b)^{2}\right]^{2}}, \\
& f_{y}=\frac{y-b}{(x-a)^{2}+(y-b)^{2}}+\frac{r(y-b)\left(\sqrt{(x-a)^{2}+(y-b)^{2}}-r\right)}{\sigma^{2}\left[(x-a)^{2}+(y-b)^{2}\right]^{2}} \text {. To solve this problem, we propose the }
\end{aligned}
$$

following method based on first order optimality condition.Solve the following equation system. Suppose that all roots are given as $\left\{\left(\bar{x}_{j}, \bar{y}_{j}\right)\right\}_{j \in J}$.

$$
\left\{\begin{array}{l}
\sum_{i=1}^{N}\left\{\frac{x-x_{i}}{\left(x-x_{i}\right)^{2}+\left(y-y_{i}\right)^{2}}+\frac{r\left(x-x_{i}\right)\left(\sqrt{\left(x-x_{i}\right)^{2}+\left(y-y_{i}\right)^{2}}-r\right)}{\sigma^{2}\left[\left(x-x_{i}\right)^{2}+\left(y-y_{i}\right)^{2}\right]^{2}}\right\}=0 \\
\sum_{i=1}^{N}\left\{\frac{y-y_{i}}{\left(x-x_{i}\right)^{2}+\left(y-y_{i}\right)^{2}}+\frac{r\left(y-y_{i}\right)\left(\sqrt{\left(x-x_{i}\right)^{2}+\left(y-y_{i}\right)^{2}}-r\right)}{\sigma^{2}\left[\left(x-x_{i}\right)^{2}+\left(y-y_{i}\right)^{2}\right]^{2}}\right\}=0 .
\end{array}\right.
$$

value $f\left(\bar{x}_{j}, \bar{y}_{j}\right)$ for different roots in $\left\{\left(\bar{x}_{j}, \bar{y}_{j}\right)\right\}_{j \in J}$. The unknown node position is given as $(x, y)=\arg \min _{j \in J} f\left(\bar{x}_{j}, \bar{y}_{j}\right)$.

\subsection{Two-dimensional search method}

According to the characteristics of equilateral triangle localization method, we further design an effective two-dimensional search method. Define the position coordinate of the unknown node $(x, y)$ as following: $\left\{\begin{array}{l}x=\sum_{i=1}^{N} \lambda_{i} x_{i}, \\ y=\sum_{i=1}^{N} \lambda_{i} y_{i} .\end{array}\right.$ where $\sum_{i=1}^{3} \lambda_{i}=1$, and, $\lambda_{i} \geq 0$. To obtain the position of the unknown nodes, we first divide the interval[0,1] into some sub-intervals with equal length. Then we calculate the objective function value corresponding to different possible position and finally we select the node with the maximal likelihood function value.

\section{The simulation results}

In this section, using the Matlab simulation platform, we do some simulation experiments and the performance comparison on the three different location methods described in this paper, RB-MML , MLE and DB-MLE.

In the simulation experiments, we select the common-used trilateration, i.e. $\mathrm{N}=3$.throngnt the results of the experiments, analyze the localization accuracy and the localization error of the three methods, and discuss the influence of different communication radius and different ranging error parameters for the average localization error, the localization precision and other performance of the methods. 
Using DR-MLE localization method, MLE localization method and conventional rang-based localization method to calculate the node location, through the simulation experiments, we get the curve diagram of the change of the location error according to the variation of $\sigma$. As shown in Figure 1.In the diagram the abscissa values is $\sigma$, the ordinate values is Relative localization error. From the graph it is easy to see that the three location methods with the increase of the ranging error parameters, the localization error was increasing.

But, in general, location error of the DR-MLE localization method are smaller than the other two kinds of methods. That is to say, this method exactly improve the location precision, and have a better localization effect in the location problem influenced by the distance factor more obviously.

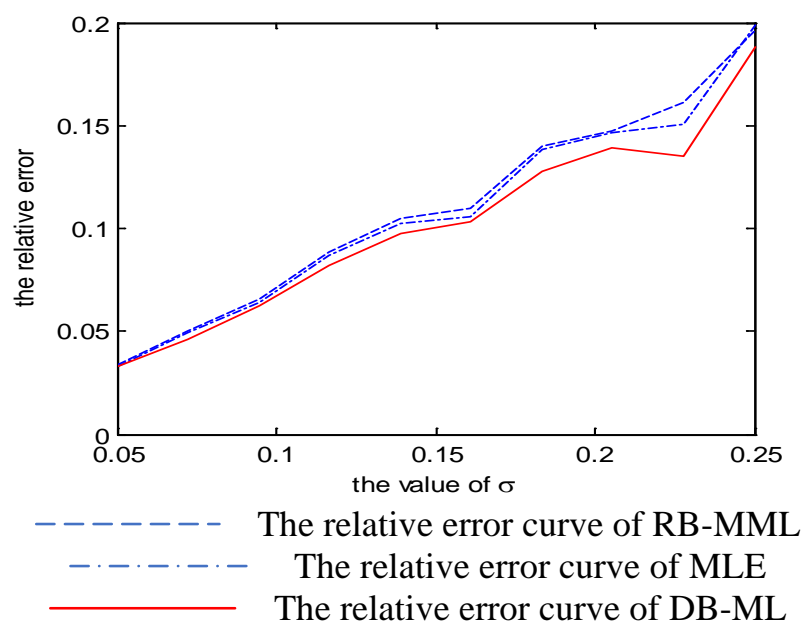

Fig.1 The variation curves of relative error of three kinds of location methods with $\sigma$ changes

\section{Conclusion}

In this paper, we propose a distance-based maximum likelihood localization estimation method for node localization in WSNs. Unlike the traditional range-based multilateral measurement localization method, which does not consider the effect of measurement errors, and the classical maximum likelihood estimation method, which suppose the measurement errors are independent of the measurement distances, the proposed DB-MLE considers the dependence of the measurement errors on the measure distances. Such assumptions are more suitable for practical distance measurement techniques, such as RSSI.

The optimization resulted from DB-MLE is a complex non-convex optimization problem. To solve it, we propose two solution methods based on the first order optimality condition and the two-dimensional search. We evaluate the performance of this proposed method through simulation experiment, and compare it with two existing localization methods. Simulation results show that DB-MLE provides higher localization accuracy compared with other methods.

\section{Acknowledgements}

This work was financially supported by Beijing Natural Science Foundation (Grant No. 4142008) , National Natural Science Foundation of China (Grant No. 61272500) , National High-tech R\&D Program (863 Program) (Grant No. 2015AA017204). Shandong Natural Science Foundation (Grant No. ZR2013FQ024).

\section{References}

[1] Jiang, N.; Jin, S.; Guo, Y., "Localization of Wireless Sensor Network Based on Genetic Algorithm,” International Journal of Computers Communications and Control, vol. 2, no. 10, pp. 184-201,2009. Vol. 8, no. 6, pp. 825-837, 2013. 
[2] J. C. Augusto, J. Liu, P. Mc Cullagh, H. Wang, and J.-B. Yang,"Management of uncertainty and spatio-temporal aspects for monitoring and diagnosis in a smart home," International Journal of Computational Intelligence Systems, vol. 1, no. 4, pp.361-378, 2008.

[3] S.-K.Yangand K.-F.Ssu, “An energy efficient protocol for target localization in wireless sensor networks,” World Academy ofScience, Engineering and Technology, vol. 56, no. 8, pp. 398-407,2009.

[4] Yu Ning, "A novel D-S based secure localization algorithm for wireless sensor networks," Security and communication networks, vol. 7, no. 11, pp. 1945-1954, 2014.

[5] R. Sugihara and R. K. Gupta, "Sensor localization with deterministic accuracy guarantee," in Proceedings of the IEEE INFO-COM, pp. 1772-1780, April 2011.

[6] Herrero, D.Martinez, H. "Range-only fuzzy Voronoi-enhanced localization of mobile robots in wireless sensor networks,” ROBOTICA, vol. 30, no. 7, pp. 1063-1077, 2012.

[7] Y. W. E. Chan and B. H. Soong, "A new lower bound on range-free localization algorithms in wireless sensor networks,” IEEE Communications Letters, vol. 15, no. 1, pp. 16-18, 2011. 